\title{
Comparison of The International Federation of Gynecology and Obstetrics (FIGO) 2018 and 2009 Staging Systems for Operated Cervical Carcinoma
}

\author{
(1) Fatma SERT, (1) Beril BALCI, (1) Senem ALANYALI, (1) Zeynep ÖZSARAN
}

Department of Radiation Oncology, Ege University Faculty of Medicine, Izmir-Turkey

\begin{abstract}
OBJECTIVE
The purpose of the study was to compare FIGO 2009 and 2018 uterine cervical carcinoma staging systems in terms of patient distribution and efficacy in predicting treatment outcome in patients treated with surgery and adjuvant radiotherapy (RT) + /-concomitant chemotherapy (CT).

\section{METHODS}

The records of 184 uterine cervical cancer patients treated with post-operative RT/RCT from 2007 to 2017 were retrospectively reviewed. Surgical procedure was in the form of Wertheim surgery in 96 patients (52\%) and pelvic lymph node dissection was performed in 32 patients (17.5\%). One hundred and sixty-six patients (87.5\%) received both external RT and intracavitary brachytherapy, and $23(12.5 \%)$ patients were treated only with external RT.

\section{RESULTS}

The median follow-up time was 61.5 months (range, 8-132 moths). One hundred and fifty-one (82\%) patients were alive, and $144(94 \%)$ of these were free of disease at the time of this analysis. The median time for locoregional failure and distant metastasis were 25 months (range, 8-88 months) and 38 months (range, 12-118 months). Stage migration was recorded in 130 patients (70.7\%) in our series when the International Federation of Gynecology and Obstetrics (FIGO) 2018 staging system was used. The most remarkable stage migration was detected for Stage I patients. A total of 119 (64.6\%) patients with Stage I showed stage migration. Five-year locoregional control, disease-free survival, disease-specific survival, and overall survival rates were $91 \%, 88 \%, 91 \%$, and $83 \%$, respectively.
\end{abstract}

\section{CONCLUSION}

The updated FIGO staging system for invasive cervical cancer incorporates imaging and pathological findings. Our results suggest us that the major improvement of 2018 staging system for uterine cervical carcinoma is that it produced better discrimination in terms of survival outcome in patients with lymph node metastases both pelvic and para-aortic.

Keywords: Cancer staging; FIGO staging; radiotherapy; uterin cervical carcinoma.

Copyright $\odot$ 2021, Turkish Society for Radiation Oncology

\section{Introduction}

In cancer treatment, staging is an essential tool for comparison of therapeutic outcomes, classification of prognostic factors, and deciding treatment. The International Federation of Gynecology and Obstetrics (FIGO) staging has played a central role all over the world providing easier comparisons of treatment 
results and aiding to make diagnosis and planning treatment for gynecologic malignancies. In 1970, FIGO introduced the first staging system for cervical uterine cancer.[1] Until now, the FIGO staging was based mainly on clinical examination with the addition of certain procedures that were allowed by FIGO to change the staging. In 2018, this has been revised by the FIGO Gynecologic Oncology Committee to allow radiological and pathological findings, where available, to assign the stage. The revised staging was presented at the FIGO XXII World Congress of Gynecology and Obstetrics. [2] Table 1 summarizes the changes in uterine cervical cancer (UCC).

Staging according to the previous systems was inadequate; with $20-40 \%$ of Stage IB-IIIB cancers understaged and up to $64 \%$ of Stage IIIB cancers overstaged.[3-5] They did not include assessment of lymph node metastases (LNM), an important determinant for prognosis and treatment planning. Moreover, radical trachelectomy, an emerging fertility-preserving technique in which the uterine corpus is anastomosed to the vagina to treat many women diagnosed during their reproductive years, was not a consideration with these previous staging systems.[6] The revisions introduced in the 2018 FIGO staging system are intended to address the gap between the staging formalism and routine clinical practice and to explicitly acknowledge the role that advanced imaging has come to play in the care of women with cervical uterine cancer. [7] In addition, new staging system clearly reflects the importance of LNM as a major prognostic factor in UCC.

After the change of staging system, all authorities expected to see a significant advance to stratify patients with different prognosis. In the perspective of new changes in staging system, we compared FIGO 2009 and 2018 staging systems in patients with operated UCC receiving adjuvant radiotherapy $+/$-chemotherapy $(\mathrm{RT}+/-\mathrm{CT})$ in our RT center.

\section{Materials and Methods}

We evaluated the medical files of UCC patients treated at the Ege University, Department of Radiation Oncology between January 2008 and December 2017. All patients underwent surgery before RT. The surgical procedure was usually total abdominal hysterectomy with bilateral salpingo-oophorectomy (TAH-BSO) in addition to pelvic and/or para-aortic lymph node dissection (LND), and partial or total omentectomy with peritoneal washing cytology. Patients with metastatic disease and patients receiving definitive RT were excluded from this analysis. All patients were staged according to both 2009 and 2018 staging systems.

Post-operative RT+CT was applied in patients with LNM, positive and/or close surgical margin, bulky tumor $(>4 \mathrm{~cm})$, and parametrial involvement, whereas post-operative RT was applied to patients with suboptimal surgery and/or with lymphovascular space involvement. Weekly concomitant cisplatin with the dose of $40 \mathrm{mg} / \mathrm{m}^{2}$ was administered to patients received concomitant CT with RT. RT consisted of external RT and intracavitary brachytherapy. External RT was applied with 6-18 MV linear accelerators to all patients. External RT was applied with 1.8 Gy daily fractions with a median total dose of 45 Gy (45-59.4 Gy). Brachytherapy was applied with high-dose rate afterloader microSelectron device to $0.5 \mathrm{~cm}$ depth from mucosal surface. Brachytherapy dose and fractionation were $3 \times 600 \mathrm{cGy}$.

Patients were followed with physical and gynecological examination, pap smear, and laboratory tests at 3 -month intervals for the first 2 years, at 6-month intervals for 3 years, and annually thereafter. For survival endpoints - locoregional control (LRC), progressionfree survival (PFS), disease-specific survival (DSS), and overall survival (OS)-time interval was defined as the time from surgery to the date of first relapse, diseasespecific death, or any death.

Table 1 Changes in cervical cancer staging system in new FIGO 2018

Stage

IB1

IB2

IB3

IIIC1

IIIC2
Previous FIGO system

Tumors with $\leq 4 \mathrm{~cm}$ size

Tumors with $>4 \mathrm{~cm}$ size
2018 FIGO system

Tumors with $<2 \mathrm{~cm}$ size

Tumors with $2-3.9 \mathrm{~cm}$ size

Tumors with $\geq 4 \mathrm{~cm}$ size

Only the patients with pelvic lymph node metastasis (either radiographic or pathologic)

The patients with paraaortic lymph node metastasis (either radiographic or pathologic) 
Statistical analysis was performed using SPSS version 21.0 (SPSS, Chicago, Illinois). The Kaplan-Meier method was performed for OS, and PFS rates and the survival curves were compared by non-parametric survival analysis (log-rank test). All time-related events (failure or death) were calculated from the diagnosis to last follow-up or death. $\mathrm{P}<0.05$ was considered statistically significant.

\section{Results}

The medical records of 184 eligible patients with histologically confirmed UCC were evaluated. The median age was 49 years (range, 18-80 years). The most commonly seen histopathologic diagnosis was squamous cell carcinoma (SCC) in 129 (70\%) patients. Other subtypes were as follows: Adenocarcinoma (17\%), adenosquamous (9\%), clear cell (3\%), and undifferentiated (1\%). Surgical procedure was in the form of Wertheim surgery in 96 patients (52\%) and TAH-BSO only in 26 patients (14\%). Pelvic LND was performed only in 32 patients (17.5\%), and pelvic lymph node (LN) sampling was performed in 30 patients (16.5\%). The median and mean number of LNs dissected were 18 and 19, respectively. One hundred and sixty-six patients $(87.5 \%)$ received both external RT and intracavitary brachytherapy, and $23(12.5 \%)$ patients were treated only with external RT. The median external pelvic RT dose was $45 \mathrm{~Gy}$ (range, 45-59.4 Gy), and median para-aortic dose was 45 Gy. One hundred and six (57.6\%) patients received concomitant weekly cisplatin with median 3 courses. Patient and treatment characteristics are listed in Table 2.

The median follow-up time was 61.5 months (range, 8-132 moths). One hundred and fifty-one (82\%) patients were alive, and 144 (94\%) of these were free of disease at the time of this analysis. One hundred and sixty-four patients were in the routine follow-up until the time of death or the last follow-up visit. The other $20(11 \%)$ patients, who were lost to follow-up, were received from general citizenship information systems and/or phone calls. Forty patients were alive with disease at their last follow-up records: 10 with pelvic wall, 9 with vaginal cuff, 13 with distant metastasis, and 8 with both lymphatic and distant metastasis. The median time for locoregional failure and distant metastasis were 25 months (range, 8-88 months) and 38 months (range, 12-118 months).

Stage migration was recorded in 130 patients (70.7\%) in data when FIGO 2018 staging system was used. There was not seen any stage change for the patients with Stage IIIB and IVA in compatible with stag-

\begin{tabular}{|c|c|c|}
\hline Characteristic & $\mathbf{n}$ & $\%$ \\
\hline \multicolumn{3}{|l|}{ Age (year) } \\
\hline Median, Range & 49 & $18-80$ \\
\hline \multicolumn{3}{|l|}{ Menopause status } \\
\hline Premenopause & 96 & 52 \\
\hline Postmenopause & 88 & 48 \\
\hline \multicolumn{3}{|l|}{ Histopathology } \\
\hline Epidermoid carcinoma & 129 & 70 \\
\hline Non-epidermoid carcinoma & 55 & 30 \\
\hline Adenocarsinoma & 32 & 17.4 \\
\hline Others & 23 & 12.6 \\
\hline \multicolumn{3}{|l|}{ Tumor size } \\
\hline$<2 \mathrm{~cm}$ & 40 & 21.7 \\
\hline $2-4 \mathrm{~cm}$ & 83 & 45.1 \\
\hline$\geq 4 \mathrm{~cm}$ & 61 & 33.2 \\
\hline \multicolumn{3}{|l|}{ Lymphovascular Invasion } \\
\hline$(+)$ & 130 & 70.7 \\
\hline$(-)$ & 54 & 29.3 \\
\hline \multicolumn{3}{|l|}{ Deep stromal Invasion } \\
\hline$(+)$ & 145 & 78.8 \\
\hline$(-)$ & 39 & 21.2 \\
\hline \multicolumn{3}{|l|}{ Parametrial Invasion } \\
\hline$(+)$ & 13 & 7 \\
\hline$(-)$ & 171 & 93 \\
\hline \multicolumn{3}{|l|}{ Vaginal Involvement } \\
\hline$(+)$ & 18 & 10 \\
\hline$(-)$ & 166 & 90 \\
\hline \multicolumn{3}{|l|}{ Surgery } \\
\hline \multicolumn{3}{|l|}{ Wertheim } \\
\hline TAH+BSO+Lymphadenectomy & 96 & 52.2 \\
\hline Suboptimal (TAH+BSO/TAH+USO/ & 62 & 33.7 \\
\hline TAH/other types of surgery) & 26 & 14.1 \\
\hline \multicolumn{3}{|l|}{ Surgical margin } \\
\hline Negative & 101 & 55 \\
\hline Positive & 83 & 45 \\
\hline \multicolumn{3}{|l|}{ LND } \\
\hline None & 25 & 13.6 \\
\hline Pelvic & 83 & 45.1 \\
\hline Pelvic+Paraaotic & 76 & 41.3 \\
\hline \multicolumn{3}{|l|}{ Nodal status } \\
\hline Pelvic positive & 40 & 21.7 \\
\hline Paraaortic positive & 6 & 3.3 \\
\hline Pelvic+paraaotic positive & 5 & 2.7 \\
\hline
\end{tabular}

TAH+BSO: Total abdominal hysterectomy with bilateral salpingooophorectomy; USO: Unilateral salpingo-oopherectomy; LND: Lymp node dissection

ing systems. The most remarkable stage migration was detected for Stage I patients. A total of 119 (64.6\%) patients with Stage I showed stage migration. About $47.8 \%$ of this migration was seen within Stage I itself according to new FIGO 2018 staging system. The remaining 
31 patients (16.8\%) with Stage I disease were upstaged to Stage IIIC disease. In addition, 11 patients $(6 \%)$ with Stage II were upstaged to Stage IIIC with new staging system (Table 3 ). We compared the stage migration in terms of histopathology (SCC vs. non-SCC). However, we could not find any difference between the stage migration and the histopathology of patients ( $\mathrm{p}>0.05)$.

Five-year LRC, PFS, DSS, and OS rates were $91 \%$, $88 \%, 91 \%$, and $83 \%$, respectively. In Table 4 , OS and

\begin{tabular}{|c|c|c|}
\hline Table 3 & $\begin{array}{l}\text { The patien } \\
\text { staging sys }\end{array}$ & $\begin{array}{l}\text { ata restaged with new FIGO } 2018 \\
\text { m }\end{array}$ \\
\hline Stage & $\begin{array}{c}\text { FIGO } 2009 \\
\text { n (\%) }\end{array}$ & $\begin{array}{l}\text { FIGO } 2018 \\
\text { n (\%) }\end{array}$ \\
\hline IA2 & $4(2.2)$ & $\begin{array}{l}1 \text { (0.5) } \\
1 \text { upstaged to 3C } \\
2 \text { upstaged to } 1 \mathrm{~B} 1\end{array}$ \\
\hline IB1 & $100(54.3)$ & $\begin{array}{l}27 \text { (14.7) } \\
54 \text { upstaged to } 1 \mathrm{~B} 2 \\
21 \text { upstaged to } 3 \mathrm{C}\end{array}$ \\
\hline IB2 & $42(22.8)$ & $\begin{array}{l}54 \text { (29.3) } \\
\quad 33 \text { upstaged to } 1 \mathrm{~B} 3\end{array}$ \\
\hline IB3* & - & $33(17.9)$ \\
\hline IIA & $25(13.6)$ & $\begin{array}{l}18 \text { ( } 9.8) \\
\quad 7 \text { upstaged to } 3 C\end{array}$ \\
\hline IIB & $9(4.9)$ & $\begin{array}{l}5(2.7) \\
\quad 4 \text { upstaged to } 3 C\end{array}$ \\
\hline IIIB & $1(0.5)$ & $1(0.5)$ (There is no stage migration) \\
\hline$I I I C^{* *}$ & - & $\begin{array}{l}42(22.8) \\
3 \text { of them were detected } \\
\text { radiologically. } \\
39 \text { of them were detected } \\
\text { pathologically. }\end{array}$ \\
\hline IVA & $3(1.6)$ & 3 (1.6) (There is no stage migration) \\
\hline
\end{tabular}

FIGO: International Federation of Gynecology and Obstetrics disease-free survival (DFS) rates according to both FIGO 2009 and FIGO 2018 were shown. The first thing should be said that all the treatment outcomes were observed excellent with both staging system. The remarkable point in this table was that the survival rates of both Stages I and II were increased due to the fact that the patients with LNM were staged as IIIC. As a result of this, treatment outcomes of both Stages I and II were detected higher in FIGO 2018.

FIGO 2018 divided Stage IB into three subgroups according to tumor size.[2] Overall and PF survival curves were similar for Stage IB2 and IB3 in new FIGO 2018 staging system. Stage IBI had better OS curve than the others in both staging systems (Fig. 1). On the other hand, PFS curve of Stage IBI was not better than the others (Fig. 1). It could be argued that as followup time became longer, patients with Stage IB3 disease would show decreased treatment outcomes.

LN metastasis has been a new parameter in UCC staging with FIGO 2018 due to known worse treatment outcome of it.[2] Small tumors with LNM upstaged to Stage IIIC.

In this current study, pelvic +/- para-aortic lymphatic dissection was performed in 159 (86.4\%) patients and para-aortic lymphatic dissection was added to pelvic lymphatic dissection in $76(41.3 \%)$ patients. The detecting way of LNM has not made any change in new staging system. In other words, lymphatic metastasis could be shown by pathologically or by radiologically. In our series, we had only three radiologic Stage IIIC patients. We detected pelvic LNM in $40(21.7 \%)$ and para-aortic LNM in $6(3.3 \%)$ patients. Totally, 46 (25\%) patients had LNM, 43 of them were pathologically detected, in our data.

We compared the OS and PFS rates in terms of both pelvic (Stage IIIC1) and para-aortic LN metas-

Table 4 Five-year overall, local recurrence-free, disease-free, and metastasis-free survival rates of patients according to both FIGO 2009 and FIGO 2018 staging systems

\begin{tabular}{|c|c|c|c|c|c|c|c|c|c|c|}
\hline \multirow[t]{2}{*}{ Stage } & \multicolumn{5}{|c|}{ FIGO 2009} & \multicolumn{5}{|c|}{ FIGO 2018} \\
\hline & n (\%) & $\begin{array}{c}\text { 5-y OS } \\
(\%)\end{array}$ & $\begin{array}{c}\text { 5- LRFS } \\
(\%)\end{array}$ & $\begin{array}{c}\text { 5-y DFS } \\
\text { (\%) }\end{array}$ & $\begin{array}{c}\text { 5-MFS } \\
(\%)\end{array}$ & n (\%) & $\begin{array}{c}\text { 5-y OS } \\
(\%)\end{array}$ & $\begin{array}{c}\text { 5-LRFS } \\
(\%)\end{array}$ & $\begin{array}{c}\text { 5-y DFS } \\
\text { (\%) }\end{array}$ & $\begin{array}{c}\text { 5-MFS } \\
(\%)\end{array}$ \\
\hline IB1 & $100(54.3)$ & 83.6 & 92.1 & 82.3 & 83.0 & $27(14.7)$ & 92.0 & 96.1 & 90.1 & 87.8 \\
\hline IB2 & $42(22.8)$ & 82.6 & 87.9 & 80.5 & 81.4 & $54(29.3)$ & 88.0 & 94.2 & 85.3 & 83.6 \\
\hline $\mathrm{IB} 3^{*}$ & - & - & - & - & - & 33 (17.9) & 81.0 & 91.3 & 75.4 & 81.2 \\
\hline IIA & 25 (13.6) & 81.0 & 81.5 & 75.6 & 78.1 & $18(9.8)$ & 85.0 & 85.2 & 80.3 & 80.1 \\
\hline IIB & $9(4.9)$ & 77.8 & 62.5 & 74.5 & 77.5 & $5(2.7)$ & 76.3 & 80.0 & 73.1 & - \\
\hline$I I I C^{* *}$ & - & - & - & - & - & $42(22.8)$ & 73.0 & 70.4 & 68.7 & 62.9 \\
\hline
\end{tabular}

***: New FIGO stages. Stages IA2, IIIB, and IVA were not included in statistical analysis due to small numbers. FIGO: International Federation of Gynecology and Obstetrics 

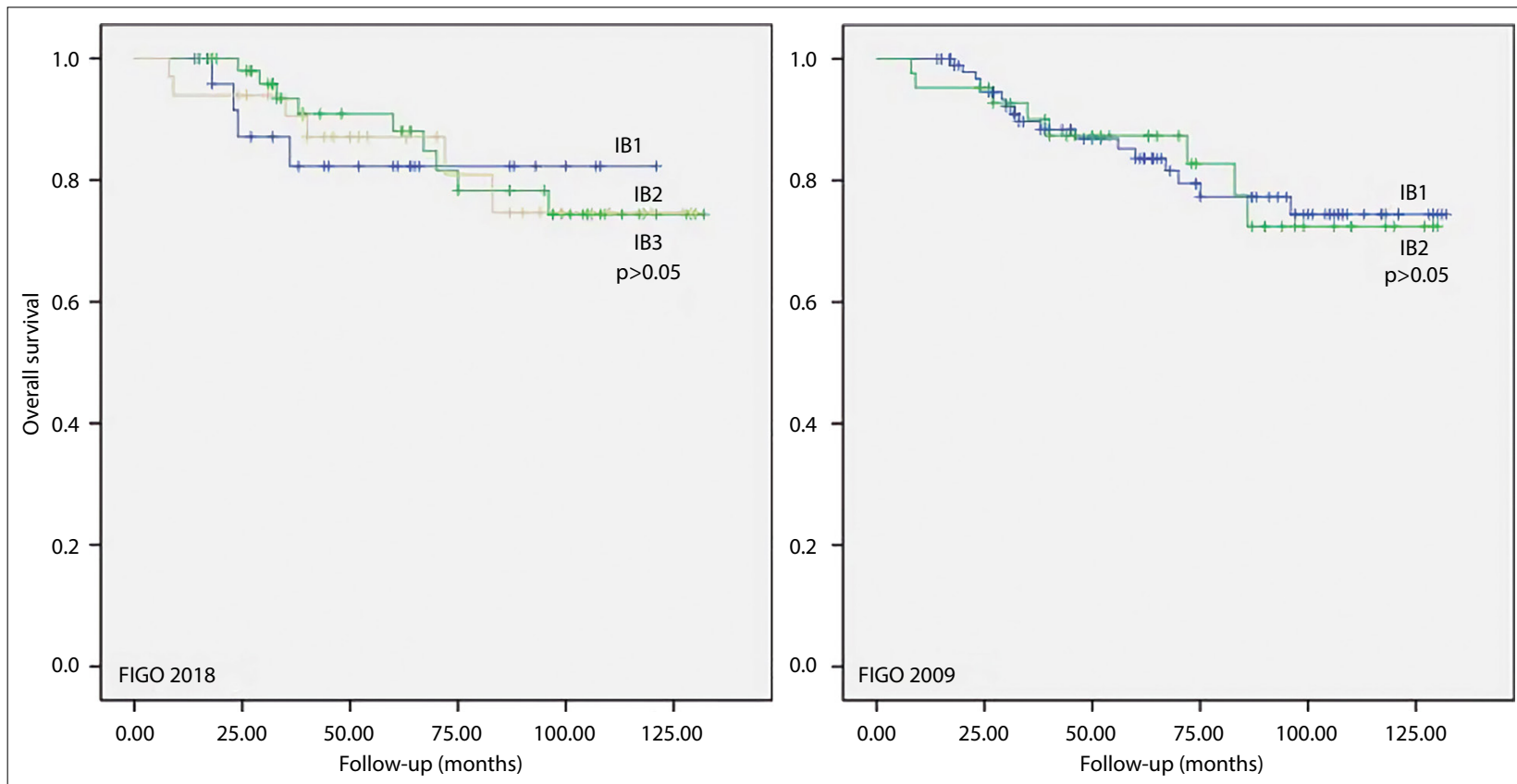

Fig. 1. Kaplan-Meier overall and progression-free survival curves for Stage IB tumors.

FIGO: International Federation of Gynecology and Obstetrics.

tasis (Stage IIIC2). Five-year OS and PFS rates were $75.1 \%$ and $61.7 \%$ for the patients with pelvic LNM (Stage IIIC1). We could not show statistically significant difference for OS rates for pelvic LNM $(75.1 \%$ vs. $85.9 \%, \mathrm{p}=0.183)$. On the other hand, PFS rates were affected unfavorably with pelvic LNM (61.7\% vs. $83.6 \%$, $\mathrm{p}=0.004$ ) (Fig. 2). Five-year OS and PFS rates were $44.4 \%$ and $50.0 \%$ for the patients with para-aortic LNM (Stage IIIC2). Both OS (44.4\% vs. $84.4 \%, \mathrm{p}=0.006$ ) and PFS (50.0\% vs. $84.1 \%, \mathrm{p}=0.001)$ rates were significantly affected by para-aortic LNM (Fig. 3).

We grouped patients according to stage migration as: (1) Within Stage I, (2) Stage II upstaged to stage IIIC, and (3) Stage I upstaged to Stage IIIC. The Kaplan-Meier plot for stage migration showed discriminative survival curves for upstaging to Stage IIIC disease. In accordance with literature, the patients had stage migration within Stage I had significantly better OS and PFS rates than the others, with 5-year OS and PFS rates of $87.7 \%$ and $96.5 \%$, respectively. The worse treatment results were detected the patients with Stage II upstaged to Stage IIIC disease in terms of both OS and PFS rates, with 5-year OS and PFS rates of $62.3 \%$ and $60.6 \%$, respectively (Fig. 4 ).

\section{Discussion}

Globally, cervical cancer continues to be one of the most common cancers among females, being the fourth most common after breast, colorectal, and lung cancer. In 2012, it was estimated that there were approximately 527.600 new cases of cervical cancer with 265.700 deaths annually. [8] In low- and middle-income countries, it is more common, being the second most common cancer in incidence among women and the third most common in terms of mortality.[9] Recent developments in imaging technology, radiotherapeutic approaches, biological target therapy, and increased use of minimally invasive surgery have drastically changed the paradigm for the management of women with cervical cancer. Until now, the FIGO staging system was based primarily on clinical examination with limited additional diagnostic procedures allowed by the FIGO staging system. $[7,10]$

There is only one recent report which could be found in literature regarding new FIGO 2018 staging system. [11] This report aimed to validate the revised FIGO 2018 staging system for cervical cancer, with a particular focus on Stage IB and Stage III disease. Based on this validation analysis of Matsuo et al. using the National Cancer Institute's Surveillance, Epidemiology, and End Results (SEER) program between 1988 and 2014, the revised FIGO staging system for cervical cancer is useful to distinguish survival groups. Applying the new system, Stage IB1 and Stage IB2 disease have distinct characteristics and outcomes. It has been shown that Stage IB1 disease is more likely to be low grade and has adenocarcinoma histology, whereas Stage IB2 dis- 

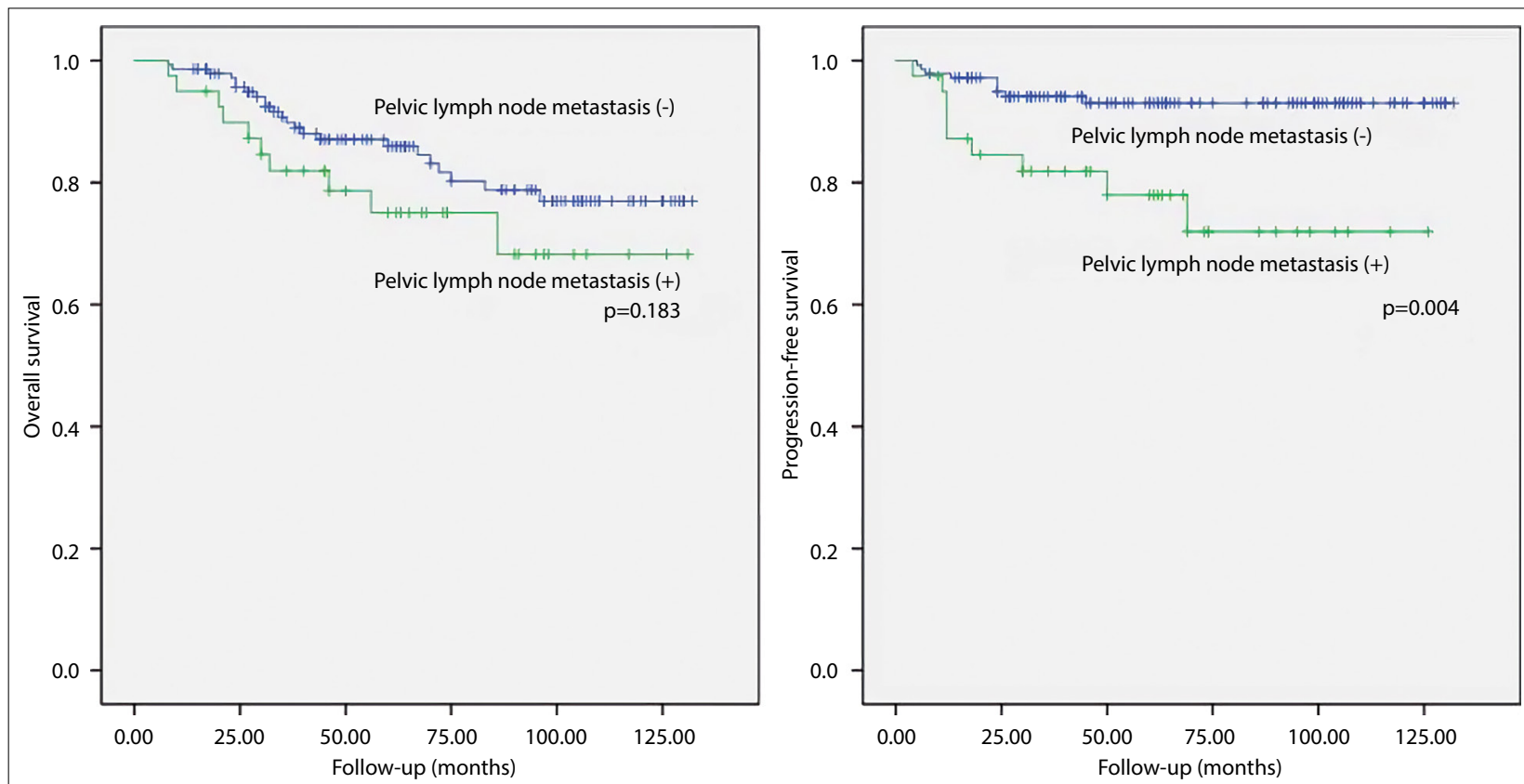

Fig. 2. Kaplan-Meier survival curves according to pelvic lymphatic involvement.
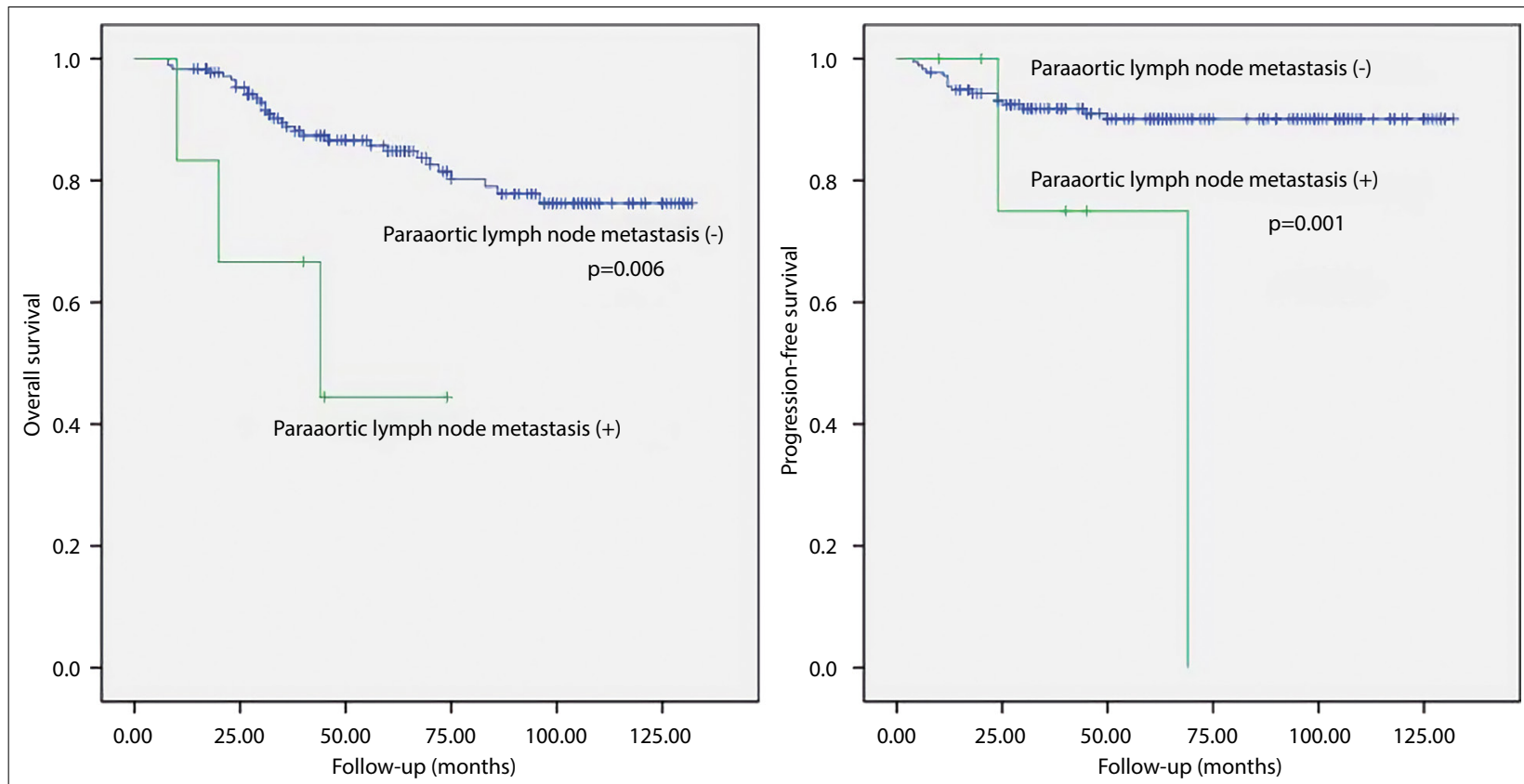

Fig. 3. Kaplan-Meier survival curves according to para-aortic lymphatic involvement.

ease is more likely to be high grade and has squamous histology.[11] We could not show any relation between histopathological tumor type and FIGO staging in our cohort. In addition, they concluded that patients with Stage IB2 disease have a nearly 2 -fold increased risk of cervical cancer death compared to those with Stage IB1 disease. Similar with this, we detected that Stage IBI had better OS curve than the others in both staging system. On the other hand, we could not show statistically significant difference in terms of treatment outcomes between new IB staging groups. By the help of focusing new predictive molecular and/or genetic biomarkers, 

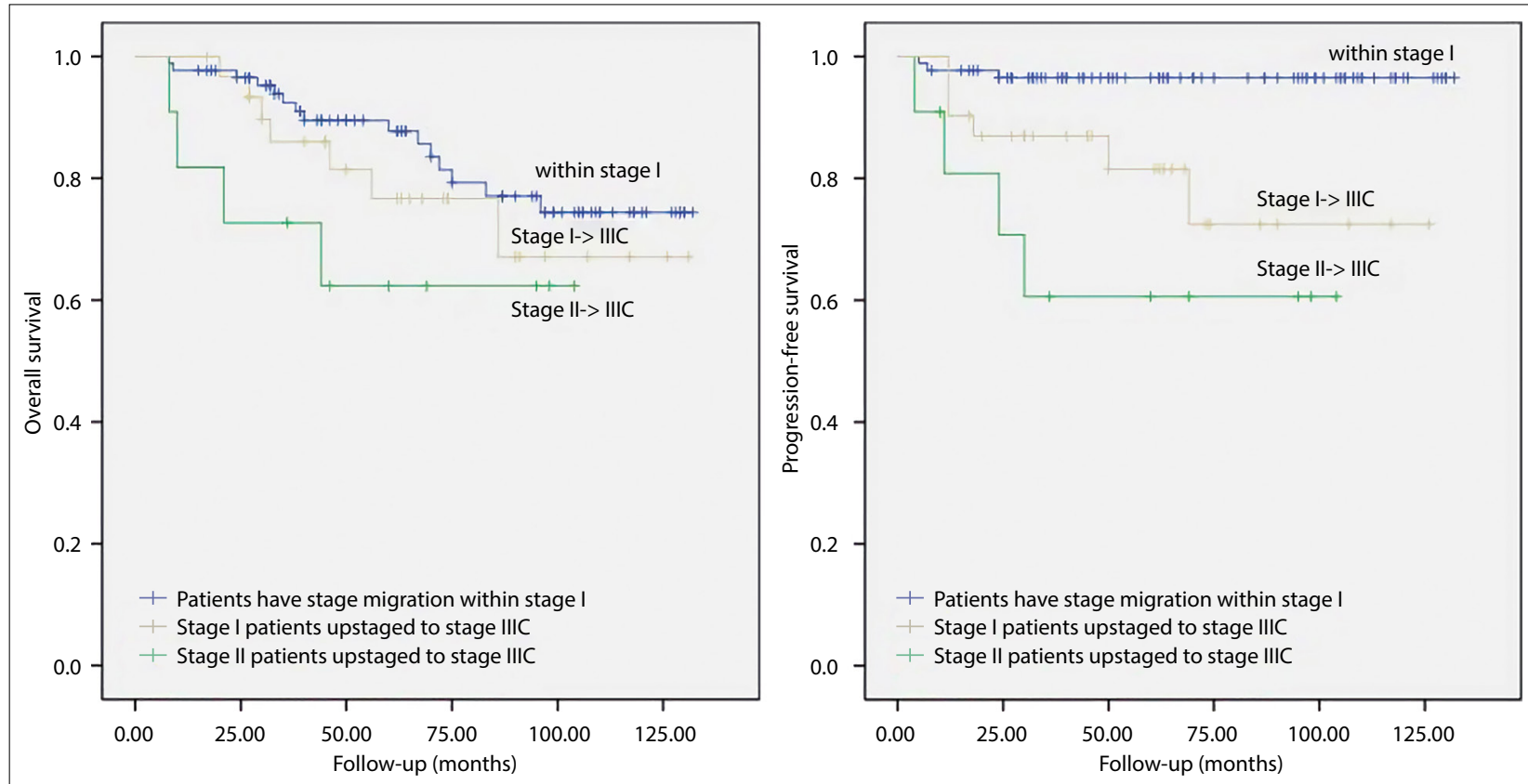

Fig. 4. Kaplan-Meier survival curves according to stage migration.

we might present new factors for distinguishing Stage IB disease rather than tumor size.

Another major change in the FIGO 2009 staging system is the inclusion of LN status into Stage III disease.[2] Patients who have documented pelvic and/ or para-aortic LN metastasis are specifically staged as Stage IIIC. Under the revised system, radiographic and/or histological findings are allowed to assign Stage IIIC disease. Stage IIIC1 is designated when only pelvic LN metastasis is detected, while Stage IIIC2 is designated when para-aortic LN metastasis is documented by either method. In the validation analysis of Matsuo et al.,[11] they evaluated 11.733 women with Stage III UCC. They found that Stage IIIC1 disease was also more likely to have adenocarcinoma or adenosquamous histology and to be higher grade but have smaller tumor size (all, $\mathrm{p}<0.001$ ). However, we could not show any statistically significant difference between Stage IIIC disease and histology. In Stage III disease, survival of women with Stage IIIC1 disease is greater for those patients with Stage IIIA or Stage IIIB disease. The analysis showed 5-year cancer-specific survival rates of $46.0 \%$ for Stage IIIA disease, $42.6 \%$ for Stage IIIB disease, and $62.1 \%$ for Stage IIIC1 disease in the trial of Matsuo et al.[11] Interestingly, this current study did not have patient with Stage IIIA. In addition, we had only one patient Stage IIIB disease. Therefore, we could not compare the treatment re- sults for the patient with these Stage III disease subgroups. In our trial, we found that 5-year OS and PFS rates were $75.1 \%$ and $61.7 \%$ for the patients with Stage IIIC1 disease. It is important not to miss that Stage IIIC1 disease reflects a heterogeneous group of tumors with a wide range of survivals based on local tumor factors. Patients with metastatic para-aortic LNs are managed with concurrent CRT, and these patients would be staged as Stage IIIC2 in the new FIGO 2018 system. Kidd et al.[12] demonstrated $>35 \%$ DSS for patients with Stage IIIC2 disease. In this current data, 5-year OS and PFS rates were $44.4 \%$ and $50.0 \%$ for the patients with Stage IIIC2 disease. Some patients with supraclavicular only LN metastasis have been shown to achieve long-term DFS. With help of this knowledge, Zighelboim et al.[13] indicated that some patients who receive external beam RT and CT for limited oligometastatic disease may also have a prolonged DFS.

The FIGO classification system combines the most basic parameters of gynecologic malignancies to stratify patients according to extent of disease, to decide the better treatment modality, and to develop a common language for the future testing of cancer treatment strategies. [1] FIGO 2018 staging system examines both clinical and radiological findings, as well. Although the patient number is low compared to large multi-institutional series, the uniform risk-adapted treatment in our patients gives 

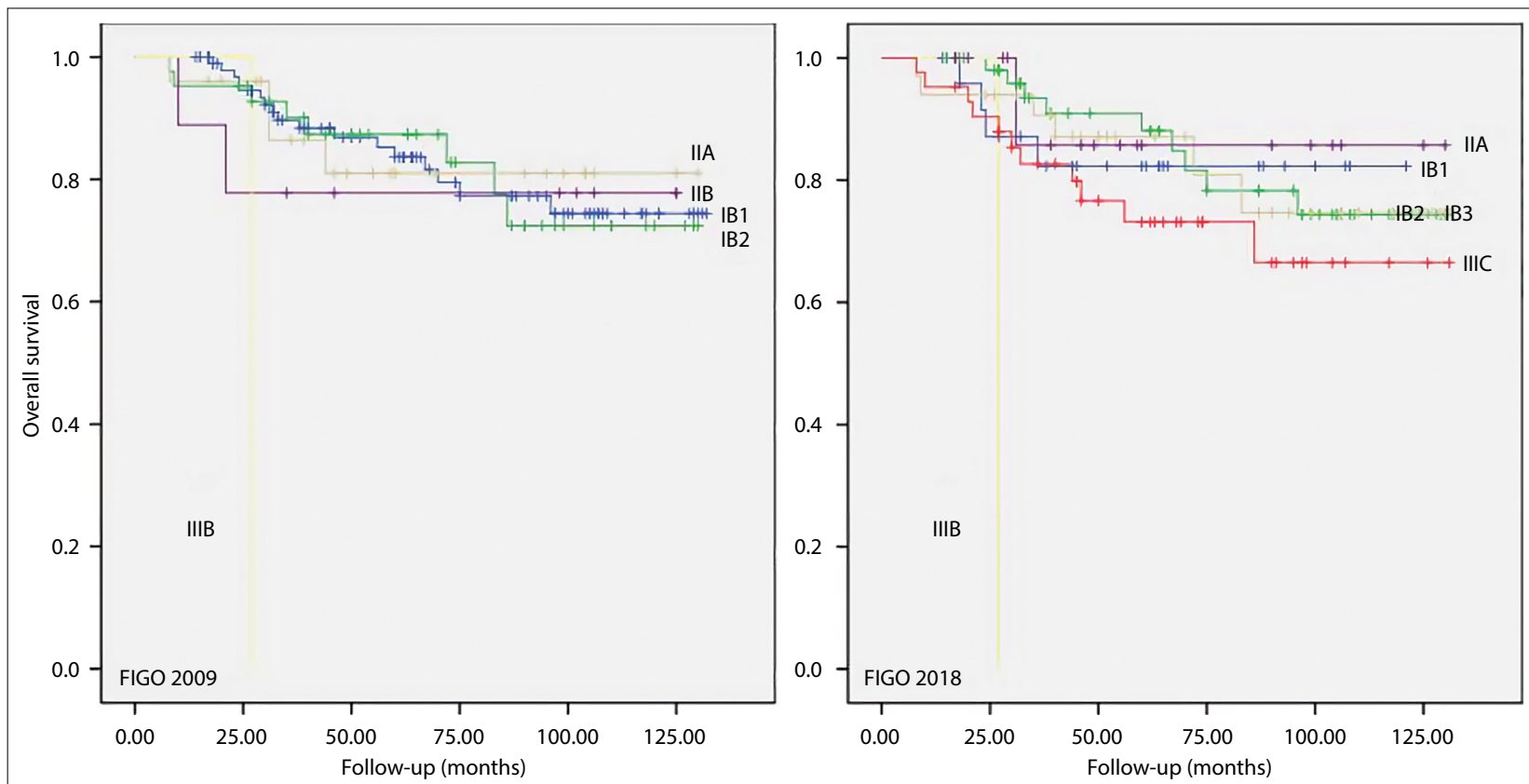

Fig. 5. Kaplan-Meier survival curves showing the stage distributions both between FIGO 2009 and FIGO 2018. FIGO: International Federation of Gynecology and Obstetrics.

us valuable information about the ability of old and new staging systems for discriminating the survival outcomes (Fig. 5). Our data showed that there were no major differences between FIGO 2009 and 2018 staging systems in terms of OS and PFS rates when comparisons were made as Stage I and II in FIGO 2009 versus 2018. Major difference was seen for the patients with Stage I and II disease upstaged to Stage III disease in terms of both OS and PFS. The worse treatment outcomes were detected for the patients with Stage II disease upstaged to Stage IIIC disease. Based on this result, it might be argued that not only LNM both also primary tumor involvement should be kept on mind while deciding treatment approach. The survival curves between stages were separated clearly with the use of FIGO 2018 (Fig. 5).

A strength of this study is that this is likely the first single institutional study to examine the comparison of the two staging system for operated UCC. Out of this current study, there is only one population-based study, done by Matsuo et al.,[11] using SEER data. Our results are important in terms of including homogenous patient data and using modern treatment techniques. On the other hand, limitation of this study includes that this is a retrospective study, and there may be missing confounding surgical pathological and follow-up evaluations.

\section{Conclusion}

The updated FIGO staging system for invasive operated UCC incorporates imaging and pathological findings. Our results suggest us that the major improvement of 2018 staging system for UCC is that it produced better discrimination in terms of survival outcome in patients with LNM both pelvic and para-aortic. In addition, the hope is that these revisions will improve the accuracy of staging and this will be reflected a more refined understanding of prognostic groups, which will facilitate better treatment for women with invasive UCC.

Peer-review: Externally peer-reviewed.

Conflict of Interest: All authors declared no conflict of interest.

Ethics Committee Approval: The study was approved by the Ege University Faculty of Medicine Clinical Research Ethics Committee (No: 18-10.2T/36, Date: 01/11/2018).

Financial Support: None declared.

Authorship contributions: Concept - F.S., B.B., S.A., Z.Ö.; Design - F.S., B.B., S.A., Z.Ö.; Supervision - F.S., B.B., S.A., Z.Ö.; Funding - F.S., B.B., S.A., Z.Ö.; Materials - F.S., B.B., S.A., Z.Ö.; Data collection and/or processing - F.S., B.B., S.A., Z.Ö.; Data analysis and/or interpretation - F.S., B.B., S.A., Z.Ö.; Literature search - F.S., B.B., S.A., Z.Ö.; Writing - F.S., B.B., S.A., Z.Ö.; Critical review - F.S., B.B., S.A., Z.Ö. 


\section{References}

1. Lee SI, Atri M. 2018 FIGO staging system for uterine cervical cancer: Enter cross-sectional imaging. Radiology 2019;292(1):15-24.

2. Bhatla N, Berek J, Cuello M. Abstract of the FIGO XXII world congress of gynecology and obstetrics. Int J Gynecol Obstet 2018;143(3):43-991.

3. Amendola MA, Hricak H, Mitchell DG, Snyder B, Chi DS, Long HJ III, et al. Utilization of diagnostic studies in the pretreatment evaluation of invasive cervical cancer in the United States: Results of intergroup protocol ACRIN 6651/GOG 183. J Clin Oncol 2005;23(30):7454-9.

4. Van Nagell JR Jr., Roddick JW Jr., Lowin DM. The staging of cervical cancer: Inevitable discrepancies between clinical staging and pathologic findinges. Am J Obstet Gynecol 1971;110(7):973-8.

5. Mayr NA, Yuh WT, Zheng J, Ehrhardt JC, Sorosky JI, Magnotta VA, et al. Tumor size evaluated by pelvic examination compared with 3-D quantitative analysis in the prediction of outcome for cervical cancer. Int J Radiat Oncol Biol Phys 1997;39(2):395-404.

6. Bhatla N, Aoki D, Sharma DN, Sankaranarayanan R. Cancer of the cervix uteri. Int J Gynecol Obstet 2018;143(2):22-36.

7. Berek JS, Matsuo K, Grubbs BH, Gaffney DK, Lee
SI, Kilcoyne A, et al. Multidisciplinary perspectives on newly revised 2018 FIGO staging of cancer of the cervix uteri. J Gynecol Oncol 2019;30(2):e40.

8. Ferlay J, Soerjomataram I, Ervik M, Dikshit R, Eser S, Mathers C, et al. GLOBOCAN 2012: Estimated Cancer Incidence, Mortality and Prevalence Worldwide in 2012 v1.0. Lyon, France: International Agency for Research on Cancer; 2013.

9. Sankaranarayanan R. Screening for cancer in lowand middle-income countries. Ann Glob Health 2014;80(5):412-7.

10. Sahdev A, Sohaib SA, Wenaden AE, Shepherd JH, Reznek RH. The performance of magnetic resonance imaging in early cervical carcinoma: A long-term experience. Int J Gynecol Cancer 2007;17(3):629-36.

11. Matsuo K, Machida H, Mandelbaum RS, Konishi I, Mikami M. Validation of the 2018 FIGO cervical cancer staging system. Gynecol Oncol 2019;152(1):87-93.

12. Kidd EA, Siegel BA, Dehdashti F, Rader JS, Mutch DG, Powell MA, et al. Lymph node staging by positron emission tomography in cervical cancer: Relationship to prognosis. J Clin Oncol 2010;28(12):2108-13.

13.Zighelboim I, Taylor NP, Powell MA, Gibb RK, Rader JS, Mutch DG, et al. Outcomes in 24 selected patients with stage IVB cervical cancer and excellent performance status treated with radiotherapy and chemotherapy. Radiat Med 2006;24(9):625-30. 